\title{
Resultados a mediano plazo del implante de stents imbricados en pacientes con infarto con supradesnivel del ST. El rol de los stents medicados
}

\author{
Marcelo Potthoff ${ }^{1}$, Gonzalo Martínez ${ }^{1}$, Martín Valdebenito $^{3}$, Luis Villarroel ${ }^{2}$, Manuel Méndez $^{1}$, Alberto Fuensalida $^{1}$, \\ Luis Quiñiñir ${ }^{4}$, Alejandro Martínez ${ }^{1}$. \\ 1. División de Enfermedades Cardiovasculares, Escuela de Medicina, Pontificia Universidad Católica de Chile. \\ 2. Departamento de Salud Pública, Escuela de Medicina, Pontificia Universidad Católica de Chile. \\ 3. Departamento de Cardiología, Cardiología intervencional, Hospital Doctor Sótero del Río. \\ 4. Departamento de Cardiología, Electrofisiología, Hospital Hernán Henriquez Aravena. \\ Investigación realizada en dependencias del Hospital Clínico, Centro de terapia endovascular, \\ Pontificia Universidad Católica de Chile y Hospital Dr Sótero del Río.
}

Introducción: El implante de dos stents imbricados (SIMB) es una práctica frecuente en el laboratorio de hemodinamia. Sin embargo, evidencia reciente sugiere que en pacientes con infarto con supradesnivel del segmento ST (IAMc/SDST) esta técnica puede asociarse a mejores resultados cuando se utilizan exclusivamente stents medicados.

Objetivo: Evaluar en una cohorte de pacientes chilenos las causas, características clínicas y sobrevida a 2 años de los pacientes con IAMc/SDST que son tratados con SIMB, en función del tipo de dispositivo y combinación utilizada; metálico/metálico (BMS/BMS), metálico/medicado (BMS/DES), medicado/medicado (DES/DES).

Método: Se realizó un estudio prospectivo, caso incidente, entre enero del año 2012 y mayo del año 2016 en nuestro laboratorio de hemodinamia. Se recolectó la información clínica, angiográfica y sobrevida a 2 años de los pacientes ingresados por IAMc/SDST que fueron tratados con SIMB. Para el análisis estadístico se utilizó chi-cuadrado, regresión logística y análisis multivariado en programa SPSS, considerando significativa una $\mathrm{p}<0,05$.

Resultados: De 2403 pacientes evaluados, el 13\% $(\mathrm{n}=312)$ recibió tratamiento con SIMB. La edad promedio fue de $68 \pm 6,6$ años y el $71 \%$ correspondió a hombres. La presencia de DMII fue de 37\%, HTA 65\%, tabaquismo $41 \%$, dislipidemia $29 \%$ y cardiopatía coronaria previa $18 \%$. Se observó que la fracción de eyec- ción promedio fue de $45 \pm 3,5 \%$, medida por método de Simpson. Las causas para imbricar stents fueron la disección post implante de stent en $72 \%$, desplazamiento de placa en $22,5 \%$ y extensión de placa más allá de la longitud del stent en un 5,5\%. El 38\% fue tratado con 2 stents BMS, el $26 \%$ con stents BMS/DES y el $36 \%$ con DES/DES. El diámetro y largo promedio de SIMB fue de $2,8 \pm 2,6 \mathrm{~mm}$ y $41,3 \pm 6,4 \mathrm{~mm}$, respectivamente. Se observó una mortalidad a 2 años de $11 \%$ en el grupo BMS/BMS, $8 \%$ BMS/DES y $6 \%$ DES/DES $(\mathrm{p}<0,01)$ y la necesidad de revascularización fue $8 \%, 5,2 \%$ y $2 \%$ respectivamente $(\mathrm{p}=0,02)$. El usar la combinación BMS/ BMS se asoció a una mayor mortalidad a 2 años en análisis de regresión logística univariado (OR 5,2, IC 95\% $2,0-17,9, \mathrm{p}<0,01)$ y multivariado (ajustado por variables clínicas, número de vasos enfermos y presentación clínica) [OR 5,5, IC 95\% 1,9-21,0, p<0,01]). Excluyendo a los pacientes con mortalidad temprana, como marcador de severidad de presentación clínica, la mortalidad a 2 años en el grupo BMS/BMS tuvo un OR de 5.9, 95\% CI 2.1- 19.5 ( $\mathrm{p}<0.01)$. No se observó diferencia en la mortalidad de pacientes tratados con 2 SIMB DES/DES y los tratados con 1 stent DES.

Conclusión: El implantar stents imbricados en pacientes con IAMc/SDST es una práctica común en el laboratorio de hemodinamia. Nuestros resultados sugieren que los resultados a mediano plazo son significativamente mejores cuando al menos uno de los stents utilizados es medicado, lo cual es concordante con reportes recientes. 


\section{Midterm results of overlapping stents implantation in patients with ST elevation Myocardial Infarction}

Background: Coronary angioplasty using two overlapping (OL) stents is a frequent practice at the cath laboratory, however the impact this strategy has on patient prognosis and the preferred stent type are largely unknown.

Aim: To evaluate 2-year outcomes of STEMI patients who underwent treatment with OL stents and assess the impact of different types of stents combinations: (BMS/BMS), (BMS/DES), or (DES/DES).

Methods: Patients presenting with STEMI undergoing primary angioplasty with 2 OL stents between January 2012 to May 2016 were included. Baseline and procedural information was collected, clinically-driven new revascularizations were recorded, and 2-year survival status was confirmed from the national database registry. OL stents technique was defined as a segment with a double layer of stents of at least $1 \mathrm{~mm}$ and less than $5 \mathrm{~mm}$ long. Statistical analyses were performed with SPSS v21.0 (IBM, Armonk, NY, USA), at with $\mathrm{p}<=0.05$ being considered significant.

Results: Of 2403 STEMI patients treated within this period, in $312(13 \%)$ the OL was used. Mean age was $68 \pm 6.6$ years. $71 \%$ males. Type 2 diabetes mellitus was present in $37 \%$, arterial hypertension in $65 \%$, smoking in $41 \%$, dyslipidemia in $29 \%$ and previously treated coronary heart disease (either CABG or PCI) in $18 \%$ of patients. Mean left ventricular ejection fraction (LVEF) as assessed by 2D Simpson method was $48 \pm 3.5 \%$. Indications for overlapping stents were plaque extension in $72 \%$, edge dissection after stent implantation in $22.5 \%$, and plaque displacement in $5.5 \%$. One, 2 or 3 vessels disease was present in $23 \%, 34 \%$ and $43 \%$, respectively. Thirty-eight percent of patients were treated with 2 BMS stents, $26 \%$ with BMS/DES stents and $36 \%$ with two DES stents. The mean stent diameter and length were $2.8 \pm 2.6 \mathrm{~mm}$ and $41.3 \pm 6.4 \mathrm{~mm}$, respectively, with no difference between the 3 groups. Post procedure target vessel revascularization was $8 \%$ for BMS/BMS, $5.2 \%$ for BMS/DES and $2 \%$ for DES/DES groups $(\mathrm{p}=0.02)$. Two-year cardiovascular mortality was $11 \%$ for the BMS/BMS group, $8 \%$ for the BMS/DES group and $6 \%$ for the DES/DES ( $\mathrm{p}<0.01$ ). BMS/BMS combination was associated with a greater cardiovascular 2-year mortality in both univariate (OR 5.2, 95\% CI $2.0-17.9, \mathrm{p}<0.01)$ and multivariate analyses ([OR $5.5,95 \%$ CI 1.9-21.0, $\mathrm{p}<0.01]$ ). After excluding early mortality cases during the first week (due to their overall severity at presentation), 2-year cardiovascular mortality in the BMS/BMS group had an adjusted OR of $5.9,95 \%$ CI $2.1-19.5(\mathrm{p}<0.01)$. There were no differences between the treatment with 2 OL DES stents and the treatment with BMS/DES.

Conclusion: Overlapping stent technique is a common practice in our cath lab, mainly driven by an initial unfavorable result with the first stent. The reported findings suggest that mid-term results are better when at least on of the imbricated stents is These results are in agreement with recent reports on the subject.

Keywords: coronary artery angioplasty, ST elevation myocardial infarction, overlapping stents. 


\section{Introducción:}

La enfermedad cardiovascular es la primera causa de muerte en países desarrollados y en vías de desarrollo ${ }^{1}$. Esto determina que en nuestro país la tasa de intervenciones coronarias percutáneas (ICP) sea de al menos 8.000 procedimientos al año ${ }^{2}$. De estas ICP, el $30 \%$ corresponde a pacientes que se presentan con infarto con supradesnivel de ST (IAMc/SDST) $)^{3}$, el grupo que concentra la mayor mortalidad y morbilidad luego de un evento coronario y que, por lo tanto, para obtener resultados favorables requiere la recanalización de la obstrucción coronaria de forma expedita, en un centro especializado y por personal capacitado $^{4}$. Durante un IAMc/SDST existen distintos grados de compromiso hemodinámico y alteraciones fisiopatológicas que dificultan la correcta interpretación de la anatomía coronaria. Se produce un estado inflamatorio sistémico, activación plaquetaria, de leucotrienos e interleukinas que determinan disfunción endotelial con distintos grados de espasmo coronario, dificultad en la visualización adecuada de la extensión de la placa ateroesclerótica y del tamaño/ diámetro del vaso a intervenir ${ }^{7}$. Dado lo anterior, en un número importante de los casos de ICP se requiere de la utilización de más de un stent coronario, teniendo que imbricar por al menos $1 \mathrm{~mm}$ un segundo stent - localizándolo en continuidad con el anterior - para un correcto tratamiento de la lesión coronaria y la restauración adecuada del flujo ${ }^{8}$. Esto se conoce como stents imbricados (SIMB)

Las causas más comunes de SIMB son i) disección del borde proximal o distal del stent posterior al implante de un primer dispositivo; ii) desplazamiento de placa más allá de los bordes del stent luego del primer implante; y iii) extensión de la placa, que no logra ser cubierta en su totalidad 9 .

Sin embargo, si bien es una técnica ampliamente utilizada, el impacto clínico que tienen los SIMB se desconoce, en particular en el contexto de IAMc/SDST. Por esto, en el este estudio se planteó como objetivo evaluar en una cohorte de pacientes chilenos las causas, características clínicas y sobrevida a 2 años de los pacientes con IAMc/ SDST que son tratados con SIMB, en función del tipo de stents utilizados.

\section{Métodos:}

Se incluyeron todos los pacientes que presentaron un IAMc/SDST y que fueron sometidos a ICP primaria durante el período comprendido entre enero del año 2012 y mayo del año 2016. Se recolectó la información clínica a través del sistema de ficha electrónica, información de la ICP primaria a través de los informes del procedimiento y angiográfica mediante análisis de los estudios realizados. Se obtuvo el estado vital de los enfermos a 2 años y la causa de muerte mediante consulta al registro civil.

Se consideró SIMB como el implante de 2 stents con una zona de solapamiento de al menos $1 \mathrm{~mm}$. Se consideró disección de los bordes del stent coronario como una división o una rotura en la pared de la arteria que comprime o compromete su lumen, reduciendo el flujo sanguíneo ${ }^{10}$ y se definió desplazamiento de placa como movimiento o aumento de volumen de placa donde previamente no se observa enfermedad en la arteria coronaria ${ }^{11}$. Se excluyó a aquellos pacientes que sólo recibieron ICP con balón como, por ejemplo, puente a cirugía cardiaca de urgencia. Los pacientes fueron agrupados en tres categorías, de acuerdo con el tipo de stents utilizados: metálico/metálico (BMS/BMS), metálico/medicado (BMS/DES), medicado/ medicado (DES/DES). Los datos son presentados como promedios \pm desviación estándar (DE) o porcentaje, según corresponda. Se utilizó chi-cuadrado para evaluar variables categóricas y T de Student para variables continuas. Se obtuvieron odds ratio mediante regresión logística y análisis multivariado. Se consideró significativa una $\mathrm{p}<0,05$. El análisis estadístico se realizó mediante el programa SPSS v21 (SPSS Inc, Chicago, IL).

\section{Resultados:}

Se incluyeron 2.403 pacientes con IAMc/SDST sometidos a ICP primaria. De ellos, el 13\% $(n=312)$ recibió tratamiento con SIMB (Tabla 1). La edad promedio fue de 68 $\pm 6,6$ años y el $71 \%$ de los pacientes correspondió a hombres. La presencia de diabetes mellitus fue de $37 \%$, hipertensión arterial $65 \%$, tabaquismo $41 \%$, dislipidemia $29 \%$ y cardiopatía coronaria previa en un $18 \%$ (incluyendo ICP y/o cirugía de revascularización miocárdica) (Tabla 2).

\begin{tabular}{|l|c|c|c|c|}
\hline \multicolumn{3}{|c|}{ Tabla 1. Características de los 3 grupos de pacientes evaluados: } \\
\hline & BMS/BMS & BMS/DES & DES/DES & Valor p \\
\hline Edad & $66 \pm 5$ & $64 \pm 3$ & $67 \pm 2$ & 0.6 \\
\hline Sexo M & $67 \%$ & $69 \%$ & $71 \%$ & 0.8 \\
\hline HTA & $58 \%$ & $66 \%$ & $63 \%$ & 0.67 \\
\hline DMII & $38 \%$ & $34 \%$ & $35 \%$ & 0.9 \\
\hline DLP & $33 \%$ & $36 \%$ & $36 \%$ & 0.7 \\
\hline TBQ & $43 \%$ & $46 \%$ & $41 \%$ & 0.6 \\
\hline Enf 1 Vaso & $25 \%$ & $29 \%$ & $28 \%$ & 0.8 \\
\hline Enf 2 vasos & $35 \%$ & $33 \%$ & $31 \%$ & 0.7 \\
\hline Enf 3 vasos & $40 \%$ & $38 \%$ & $41 \%$ & 0.6 \\
\hline
\end{tabular}




\begin{tabular}{|l|}
\hline Tabla 2. Motivo para imbricar stents: \\
\hline Lesión extensa \\
\hline Disección de bordes \\
Desplazamiento de placa \\
\hline
\end{tabular}

Enfermedad coronaria significativa (estenosis angiográfica $\geq 50 \%$ ) de 1,2 y 3 vasos se presentaron en $23 \%, 34 \%$ y $43 \%$ de los pacientes, respectivamente, y la fracción de eyección promedio fue de $45 \pm 3,5 \%$, medida en $2 \mathrm{D}$ por método de Simpson.

Se observó que el $38 \%$ de los casos fue tratado con 2 stents metálicos imbricados (BMS/BMS), el 26\% con 1 stent metálico y 1 liberador de drogas (BMS/DES) y el $36 \%$ con 2 stents liberadores de drogas DES/DES. El diámetro y largo promedio de SIMB fue de 2,8 \pm 2,6 mm y 41,3 $\pm 6,4 \mathrm{~mm}$, respectivamente. No se observó diferencias significativas respecto a los factores de riesgo clínicos y angiográficos en los 3 grupos de tipo de stents analizados. Las causas para imbricar stents fueron: disección post implante de stent en $72 \%$ de los casos, desplazamiento de placa en $22,5 \%$ y extensión de placa más allá de la longitud del stent en un 5,5\%.

Con respecto a los resultados a 2 años se identificó una mortalidad de $11 \%$ en el grupo tratado con SIMB BMS/ BMS, de $8 \%$ en el grupo BMS/DES y de $6 \%$ en el grupo DES/DES $(\mathrm{p}<0,01)$ y la necesidad de revascularización, en el mismo periodo, fue de $8 \%, 5,2 \%$ y $2 \%$ respectivamente

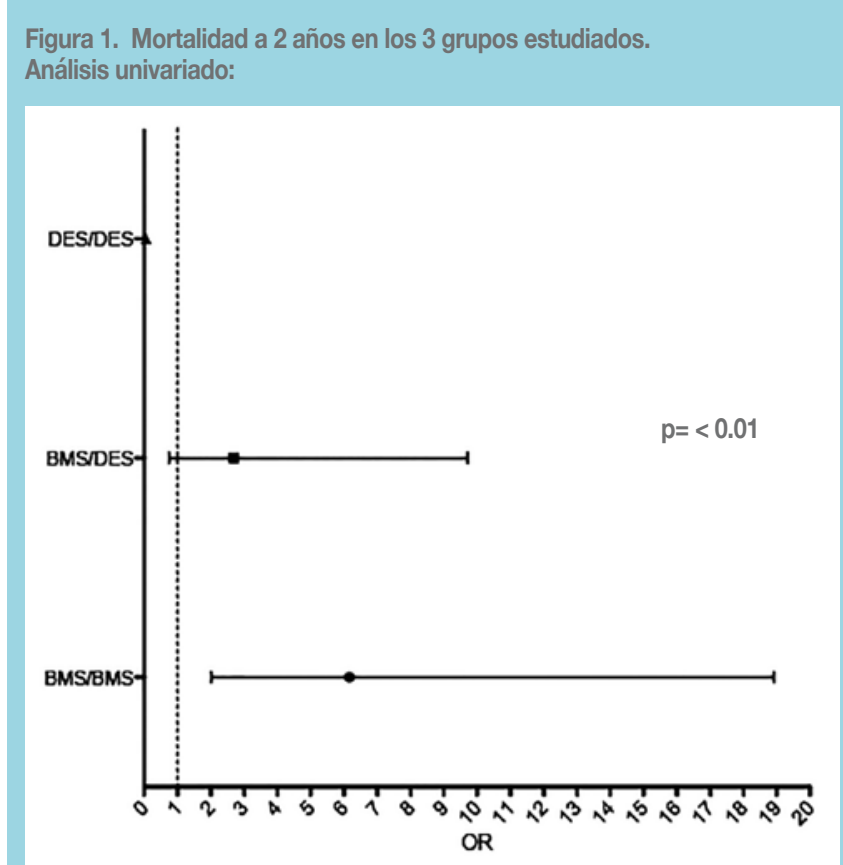

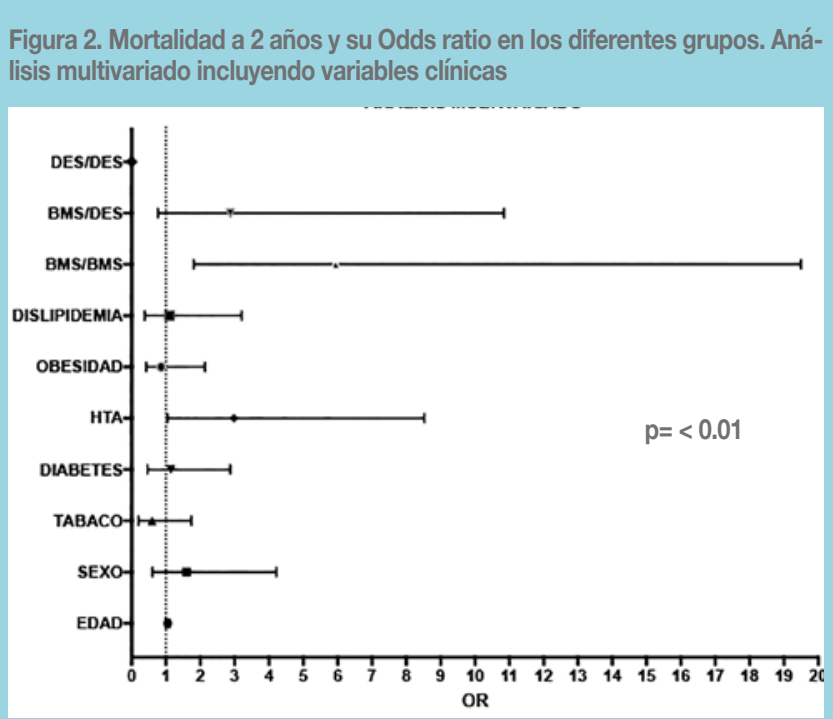

$(\mathrm{p}=0,02)$ (Tabla 3). El usar la combinación de SIMB BMS/ BMS se asoció a mayor mortalidad a 2 años en análisis de regresión logística univariado (OR 5,2, IC 95\% 2,0-17,9, $\mathrm{p}<0,01$ ) (Figura 1) como también en análisis de regresión logística multivariado (ajustado por variables clínicas, número de vasos enfermos y presentación clínica) (OR 5,5, IC 95\% 1,9-21,0, p<0,01) (Figura 2). Luego, realizamos un análisis de sensibilidad en el cual se excluyeron aquellos pacientes con mortalidad intrahospitalaria, como una forma de excluir a los enfermos que ingresaron más graves y en los cuales su pronóstico estaría más bien determinado por su presentación inicial. En este análisis, la mortalidad a 2 años mantiene su significancia en el grupo BMS/BMS con un OR de 5,9, 95\% CI 2,1- 19,5 (p<0,01).

Se debe destacar que al evaluar y comparar a los pacientes tratados con 1 stent DES versus 2 Stent DES imbricados, no se observó diferencia significativa en cuanto a mortalidad a los 2 años de evaluación $(\mathrm{p}=0,08)$.

\section{Discusión:}

El principal hallazgo de nuestro estudio es que los pacientes con IAMc/SDST que recibieron 2 stents metálicos

\begin{tabular}{|lcc|}
\hline $\begin{array}{l}\text { Tabla 3. Mortalidad y porcentaje de revascularización } \\
\text { a } 2 \text { años: }\end{array}$ & Mortalidad & Revascularización \\
\hline & $11 \%$ & $8 \%$ \\
\hline BMS/BMS & $8 \%$ & $5.2 \%$ \\
BMS/DES & $6 \%$ & $2 \%$ \\
DES/DES & 0.01 & 0.02 \\
\hline VALORP & & \\
\hline
\end{tabular}


imbricados presentaron mayor mortalidad y necesidad de reintervención en el seguimiento a 2 años. Estos resultados contrastan con los primeros reportes del uso de esta técnica con DES de primera generación (Cypher y Taxus), en los cuales se observó un aumento de la morbilidad, trombosis de stent y reinfarto con SIMB ${ }^{8,12,13}$. Por el contrario, estudios recientes con DES de última generación y liberadores de everolimus muestran al solapamiento de stents como un procedimiento seguro y eficaz. Esto está reafirmado por los hallazgos recientes del estudio EXAMINATION ${ }^{14}$, donde no se observó diferencia significativa en mortalidad, revascularización y trombosis de stent, cuando se utiliza 2 SIMB DES v/s 1 stent medicado para la resolución de lesiones durante un IAMc/SDST.

En nuestro medio el uso de BMS aún es alto, principalmente por restricciones económicas en el servicio público, de modo que los resultados aquí expuestos adquieren relevancia. Nuestros hallazgos sugieren que en casos en los cuales se decide imbricar stents, al menos uno debiera ser liberador de drogas. Del punto de vista práctico, pareciera ser una estrategia razonable que en casos en que se ha realizado una ICP primaria con un stent metálico y el operador decide que necesita posicionar un nuevo stent imbricado al anterior, este dispositivo debiera ser medicado, lo cual puede traducirse en mejores resultados a mediano plazo para el enfermo.

Es esperable que el uso de stents metálicos imbricados en un contexto donde frecuentemente se pueden sub-dimensionar los dispositivos como IAMc/SDST, con extensiones largas y diámetros pequeños, lleven a una mayor tasa de revascularización, como la aquí presentada. Sin embargo, es más difícil explicar cómo el uso de SIMB metálicos se traduce en una mayor mortalidad. En ensayos clínicos, el uso de BMS no parece asociarse a mayor mortalidad ${ }^{15}$. Es posible teorizar que, en la práctica clínica real en nuestro sistema de salud, la identificación de restenosis sea tardía y determine que los pacientes se presenten con eventos agudos y en caso de presentar dichos eventos, la respuesta del sistema sea más lenta, de modo que esto se traduzca en un compromiso de la sobrevida de los enfermos, diferente a lo que ocurre en los ensayos clínicos, altamente vigilados. Del mismo modo, es también posible que una menor intensidad de las medidas de prevención secundaria determine una progresión mayor y más rápida de la restenosis, favoreciendo eventos clínicos deletéreos. Sin embargo, los resultados aquí expuestos requieren más clarificación mediante nuevos estudios con diseños prospectivos.

Es importante destacar que en estudios publicados previamente no se describe y analiza de forma adecuada las características clínicas, angiográficas y el diámetro/largo promedio de los stents utilizados, lo que dificulta el correcto análisis de los datos presentados. En nuestro trabajo no observamos diferencias significativas con respecto a las características clínicas, angiográficas y de stents utilizados en los distintos grupos de SIMB analizados, con un diámetro/largo promedio de 2,8 $\pm 2,6 \mathrm{~mm}$ y largo $46 \pm 6,4 \mathrm{~mm}$. El presente estudio presenta una serie de limitaciones. En primer término, se realizó sólo en 2 centros de nuestro país, de modo que no representa la realidad nacional. Segundo, el número de pacientes aún es insuficiente para obtener conclusiones robustas. Tercero, es necesario realizar un estudio prospectivo, aleatorizando las distintas estrategias de combinaciones de stents para confirmar estos resultados exploratorios. Finalmente, queda pendiente establecer los mecanismos que pueden explicar nuestros hallazgos.

\section{Conclusión:}

El implantar stents imbricados en pacientes con IAMc/ SDST es una práctica común en el laboratorio de hemodinamia. Nuestro estudio sugiere que los resultados a mediano plazo son significativamente mejores cuando al menos uno de los stents utilizados es medicado. 


\section{Referencias}

1. NAZZAL C, BARTOLUCCI J, LANAS F, PRIETO JC, CORBALÁN R, CUMSILLE F. Mortalidad por infarto agudo al miocardio en tres periodos del Registro GEMI : diferencias entre hombres y mujeres. Rev Chil Cardiol 2003; 72: 172.

2. CAMPOS P, NAZZAL C, PRIETO JC, SANHUEZA P, VENEGAS J. Cumplimiento de las guías clínicas en pacientes de alto riesgo del Registro GEMI. Rev Chil Cardiol 2006; 25: 13.

3. PRIETO JC, SANHUEZA C, MARTÍNEZ N, NAZZAL C, CORBALÁN R, CAVADA G, et al . Mortalidad intrahospitalaria en hombres y mujeres según terapias de reperfusión en infarto agudo del miocardio con supradesnivel del ST. Rev Méd Chile 2008; 136: 143-50.

4. Objetivos sanitarios para la década 2000-2010. Disponible en: http://epi.minsal.cl/epi/html/elvigia/vigia15.pdf .

5. VAN DE WERF F, BAX J, BETRIU A, BLOMSTROM-LUNDQVIST C, CREA F, FALK V, et al. Management of acute myocardial infarction in patients presenting with persistent ST-segment elevation: the Task Force on the Management of ST-Segment Elevation Acute Myocardial Infarction of the European Society of Cardiology. Eur Heart J. 2008;29:2909-45.

6. WIDIMSKY P, FAJADET J, DANCHIN N, WIJNS W. Stent 4 Life" targeting PCI at all who will benefit the most. A joint project between EAPCI, Euro-PCR, EUCOMED and the ESC Working Group on Acute Cardiac Care. EuroIntervention. 2009;4:555-7.

7. ANTMAN, E.M., BRAUNWALD, E. Acute myocardial infarction. in: E. Braunwald, D.P. Zipes, P. Libby (Eds.) Heart Disease: A Textbook of Cardiovascular Medicine. W.B. Saunders, Philadelphia, Pennsylvania; 2001:1114-1231.

8. RABER L, JUNI P, LOFFEL L, WANDEL S, COOK S, WENAWESER P, et al. Impact of stent overlap on angiographic and long-term clinical outcome in patients undergoing drug-eluting stent implantation. J Am Coll Cardiol. 2010;55:1178-88.

9. O'SULLIVAN CJ, STEFANINI GG, RABER L, HEG D, TANIWAKI M, KALESAN B, et al. Impact of stent overlap on long-term clinical outcomes in patients treated with newer-generation drug-eluting stents. EuroIntervention. 2014;9:1076-84.

10. DASH D. Complications of coronary intervention: abrupt closure, dissection, perforation. Heart Asia. 2013;5(1):61-65. doi:10.1136/heartasia-2013-010304.

11. LEFÈVRE, T., LOUVARD, Y., MORICE, M.-C., DUMAS, P., LOUBEYRE, C., BENSLIMANE, A., et al. Stenting of bifurcation lesions: Classification, treatments, and results. Cathet. Cardiovasc. Intervent., 2000; 49: 274-283.

12. PAN M, DE LEZO JS, MEDINA A, ROMERO M, GONZALEZ S, SEGURA J. et al. Influence of stent treatment strategies in the long-term outcome of patients with long diffuse coronary lesions. Catheter Cardiovasc Interv. 2003;58:293-300.

13. KIM YH, PARK SW, LEE CW, HONG MK, GWON HC, JANG Y. et al. Comparison of sirolimus-eluting stent, paclitaxel-eluting stent, and bare metal stent in the treatment of long coronary lesions. Catheter Cardiovasc Interv. 2006;67:181-187.

14. ORTEGA-PAZ L, BRUGALETTA S, GIACCHI G, ISHIDA $\mathrm{K}$, CEQUIER A, IÑIGUEZ A, et al. Impact of stent overlapping on long-term clinical outcomes in patients with ST-segment elevation myocardial infarction: insights from the 5-year follow-up of the EXAMINATION trial. EuroIntervention 2017; Jaa-107 2017

15. SHAH A, FELDMAN DN. Outcome of the HORIZONS-AMI trial: bivalirudin enhances long-term survival in patients with ST-elevation myocardial infarction undergoing angioplasty. Vascular Health and Risk Management. 2012;8:115-123. 